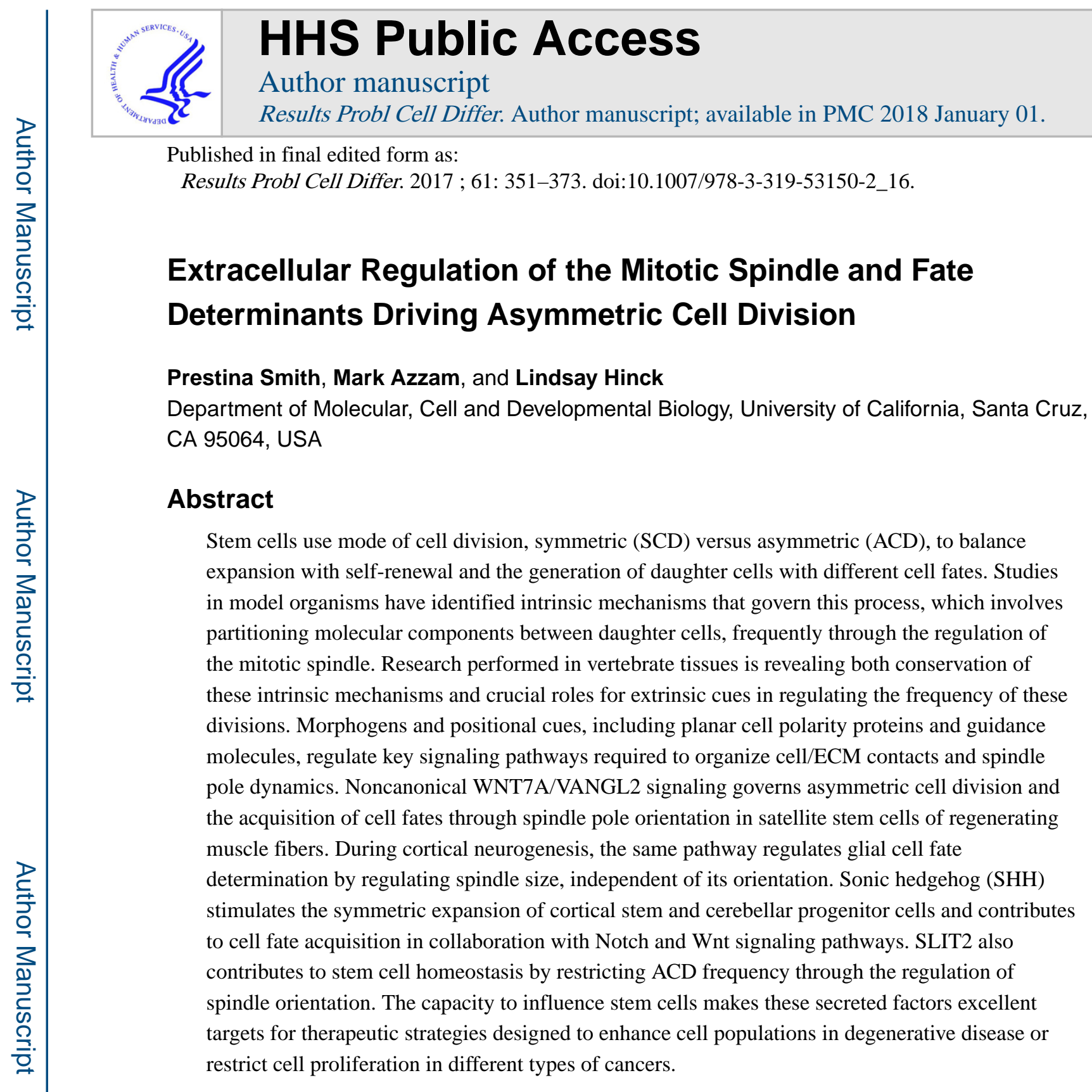

\title{
16.1 Introduction
}

Key characteristics of stem/progenitor cells are their long-term capacity to expand, selfrenew, and differentiate, attributes that serve as the foundation for the contribution of stem cells to tissue growth during morphogenesis, the maintenance of tissue homeostasis over time, and response to injury under critical circumstances. In accomplishing these tasks, stem cells undergo different modes of cell division. With asymmetric cell divisions (ACDs), stem cells self-renew, reproducing the stem cell while also generating a daughter progenitor that will adopt a different cell fate. In contrast, symmetric cell divisions (SCDs) result in identical daughters, either two stem cells or two differentiating daughter cells. 
The largest range of stem cell responses occurs when population dynamics control stem cell behavior and stem cells divide via both SCD and ACD to maintain homeostasis or respond to injury depending on intrinsic and extrinsic cues. The intrinsic ability of stem cells to drive ACD was initially defined in model organisms (Morin and Bellaiche 2011). In these systems, the intrinsic nature of cell fate specification varies among stem/progenitor cell types based on their location and history of cell contacts. For example, in studies in which individual Drosophila central nervous system progenitors were isolated and cultured, differences were observed in the capacity of cells to self-renew and generate appropriate progeny based on their origin in the embryo (Ceron et al. 2006; Luer and Technau 2009). This suggests that cells are primed for cell fate acquisition during development based on environment cues. Thus, even in model organisms, intrinsic fate determination is influenced by extrinsic factors.

Mechanisms underlying intrinsic ACD depend on the acquisition of cellular asymmetry during interphase, which is subsequently used in mitosis to polarize the distribution of proteins that determine cell fate. The mitotic spindle is reoriented in reference to this polarity axis to produce an asymmetric division. The molecular requirements for these intrinsic ACDs have been determined and include the Par3/ Par6/atypical protein kinase C complex that establishes and maintains apico-basal polarity and the microtubule-associated nuclear mitotic apparatus protein (NuMA)/LGN/Gai complex that reorients the mitotic spindle along this apico-basal axis.

The basic process of spindle reorientation is conserved in mammalian tissues; however, there is mounting evidence that the increased complexity of higher organisms generates additional regulatory requirements. This includes extrinsic mechanisms in the form of secreted cues to regulate the mode of stem and progenitor cell division. Here, we describe recent studies identifying such cues and how they govern the crucial balance between SCD and ACD. These extracellular cues govern the choice between cell expansion and differentiation during development and in response to injury.

\subsection{WNTs Function in Planar Cell Polarity}

WNTs are secreted proteins that regulate various aspects of development and signal through canonical and noncanonical pathways. The canonical pathway, which is responsible for the regulation and subcellular localization of the transcription factor $\beta$-catenin (CTNNB1), is being covered elsewhere in this volume. Here, we describe WNT signaling in governing ACD through one of the two noncanonical pathways. The second of these noncanonical pathways results in the release of intracellular calcium, which is, in turn, associated with the activation of various enzymes such as $\mathrm{Ca}^{2+} /$ calmodulin-dependent (CamKII) protein kinase and Protein Kinase C (PKC) (De 2011). This pathway has not been implicated in ACD. Instead, the noncanonical WNT pathway regulating ACD is the planar cell polarity (PCP) pathway that is activated by noncanonical WNTs (WNT7 or WNT5A). Traditionally, this pathway functions to uniformly orient cells in a sheet of epithelium by establishing proximal-distal polarity in each cell (Devenport 2014). This is achieved by regulation of both cytoplasmic and membrane proteins. Originally characterized in Drosophila and later found in mammals, the core PCP genes include Van Gogh (Vang), Flamingo (Fmi) (Celsr in 
mammals), Frizzled (Fzd), Prickled (Pk), and Disheveled (Dsh). Van Gogh and Frizzled encode multi-pass transmembrane proteins, while Disheveled and Prickled encode cytoplasmic proteins. PCP protein localization follows a stereotypic pattern in postmitotic cells and is characterized by the asymmetric localization of these core PCP proteins. Within each individual epithelial cell, VANG and PK partition to the proximal side, while FZD and DSH localize to the cell's distal region (Fig. 16.1a). At the membrane of this cell, a proximal complex forms between VANG and FMI, whereas FZD and FMI interact on the opposite membrane. Across adjacent cells, junctions form when FMI of each complex binds to the other, bringing VANG and FZD into close proximity (Devenport 2014). The result of this repetitive patterning of PCP components in sheets of epithelia is the uniform orientation of global directional cues that can be employed to produce locally polarized cell behaviors. One of these polarized behaviors occurs in mitotic cells when noncanonical extracellular WNTs regulate the orientation of cell division and consequently the fate of daughter cells through the PCP pathway. WNTs take advantage of the asymmetric localization of these core PCP proteins to drive ACD by two major interrelated mechanisms: (1) cooperation with cell fate determinants and (2) regulation of the spindle pole.

\subsubsection{Noncanonical WNT/PCP Governs the Balance Between SCD and ACD in Muscle Satellite Stem Cells}

Noncanonical WNTs have the capacity to drive ACD through the PCP pathway by regulating spindle orientation. Specifically, studies on satellite cells in adult muscle have identified a mechanism by which WNT7A, VANGL2, and FZD control ACD versus SCD in satellite stem cells by orienting the plane of cell division. Satellite cells are a wellcharacterized, mixed population of stem and progenitor cells that are present in the adult tissue and are responsible for muscle repair. They are located between the basal lamina and sarcolemmal membranes of a muscle fiber and contained within small membrane depressions (Dumont et al. 2015) (Fig. 16.2a, b). In order to maintain their number, the cells remain quiescent until activated. Once activated, a process that is initiated by either traumatic injury or daily wear and tear, muscle satellite stem cells proliferate via ACD or SCD. ACDs allow self-renewal and population maintenance, whereas SCDs generate precursor cells that undergo multiple rounds of division before terminally differentiating and fusing onto host fibers. Repeat injury models reveal a remarkable ability of these cells to balance these processes to ensure lifelong upkeep of muscle (Dumont et al. 2015). Recent studies have focused on understanding how these satellite stem cells divide via ACD to selfrenew, while producing progenitors that maintain the heterogeneity of the satellite cell population. During an ACD, satellite stem cells divide in the apical-basal plane, with one daughter cell self-renewing in contact with the basal lamina and the other daughter becoming a progenitor cell in contact with the sarcolemma. In contrast during SCD, which occurs in response to injury, the dividing satellite stem cell maintains a connection to the basal lamina; both daughters maintain their stem cell identity, resulting in expansion of the stem cell population (Dumont et al. 2015). Le Grand and colleagues have recently demonstrated that this symmetric expansion occurs via PCP signaling (Le Grand et al. 2009). PCP proteins regulate the plane of stem cell division so that both daughters maintain their contact with the basal lamina, thereby preserving their niche and supporting SCD. 
The authors investigated SCD in the satellite cell population by isolating genes specifically expressed in satellite stem cells (Le Grand et al. 2009). One identified gene was the WNT receptor $\mathrm{Fzd} 7$, and subsequent immunohistochemistry experiments on fixed muscle tissue showed FZD7 specifically expressed in a subpopulation of quiescent satellite cells. To determine whether FZD7 is regulated when satellite stem cells are stimulated, the authors injured myofibers in culture using cardiotoxin. In response to this damage, Fzd 7 was upregulated along with $W n t 7 a$, a known ligand, suggesting a role for this signaling pathway in regulating the regeneration of muscle fibers in response to injury. This result supported previous research demonstrating the expression and activity of WNTs and their FZD receptors in the satellite stem cell population during muscle regeneration (Polesskaya et al. 2003). However, these previously published studies did not address the underlying mechanism of WNT signaling in this context.

Bringing new insight into the role of WNTs in regulating satellite stem cell in response to injury, Le Grand and colleagues show that FZD7 forms a complex with co-expressed PCP pathway component, VANGL2 (Le Grand et al. 2009). To further address the intersection between noncanonical WNT and PCP signaling, the authors stimulated the proliferation of quiescent stem cells in culture using WNT7A and examined the outcome of the first division by immunostaining. They assayed both cell fate by quantifying Myf5 expression and the plane of division by investigating whether cell doublets were oriented parallel (SCD) or perpendicular (ACD) to each other with respect to the surrounding myofiber. Upon WNT7A stimulation, satellite cells divided primarily via $\mathrm{SCD}$, with spindle poles oriented parallel to the basal lamina. These dividing satellite cells also contained increased VANGL2 that was localized at opposite poles of each daughter cell (Fig. 16.2c). Loss of Fzd7 or knockdown of Vangl2 impaired the ability of WNT7A to stimulate SCDs.

Taken together, this study supports a model in which satellite cells employ a classic PCP pathway, WNT7A signaling via FZD7 to VANGL2 that controls the orientation of satellite cell division and, as a result, their cell fate within the niche. This is achieved by polarizing VANGL2 to the opposite ends of daughter cells as they undergo SCD, an organization of VANGL2 that is distinct from the planar asymmetry of VANGL2 in sheets of cells. This finding suggests that VANGL2 localizes differently, depending on whether a cell is contacted on both sides or, in the case of a lone dividing satellite stem cell, along only one side. VANGL2 is distributed to the same cellular side in cells aligned in sheet (Fig. 16.1a) but to the side of no cell contact in cells that share a single border (Fig. 16.2c). Opposite polarization of VANGL2 in a couplet of satellite stem cells ensures a continuous border with the basal lamina and preserves their localization relative to the niche (Fig. 16.2c). Thus, differential orientation of VANGL2 to distinct cell membranes generates cellular contacts required to stabilize cells in orientations that regulate the fundamental process of stem cell division.

\subsubsection{Noncanonical WNT/PCP Regulates ACD and Cell Fate Acquisition During Cortical Neurogenesis Through Spindle Size}

The ability of WNT7A to regulate ACD frequency and cell fate through the PCP pathway is not limited to the cells of the regenerating muscle. In a process called spindle size 
asymmetry, the same combination of factors also functions during corticogenesis to regulate the relative size and shape of the mitotic spindle (Delaunay et al. 2014). The cerebral cortex is stratified into layers, with each layer containing a characteristic distribution of neuronal cell types (Fig. 16.3a). During embryogenesis, many precursor cells in the cortex are responsible for generating this diverse array of differentiated cells. One of these precursors, called the apical precursor, is a major type of radial glial cell that resides along the ventricular surface (Fig. 16.3b). During cortical neurogenesis, apical precursors undergo ACD to self-renew while generating a cell fate restricted neuron. While spindle pole orientation during apical precursor ACD may provide one level of regulation (GauthierFisher et al. 2009), PCP does not appear to determine the axis of cell division in this context. Instead, PCP governs cell fate determination in these ACDs by signaling through WNT7A/ VANGL2 to regulate the position of the metaphase plate and consequently the relative size of each spindle (Fig. 16.3c). The result of this spindle size asymmetry is that the daughter cell issued from the larger spindle becomes a neuron, whereas the daughter generated from the smaller spindle maintains its status as an apical progenitor cell.

In the murine cortex, apical precursors undergo the bulk of their ACDs from embryonic day (E) E11.5-E16.5 (Delaunay et al. 2014). To track spindles in apical precursors at metaphase, Delaunay and colleagues used confocal imaging to acquire high-resolution images of spindle poles. Examining different stages of cortical neurogenesis, they found that spindle size asymmetry was initially minimal, peaked at mid-corticogenesis, and decreased at the close of this developmental period, a time course in which the peak of ACD is correlated with the highest level of spindle size asymmetry. To investigate the mechanism underlying this asymmetry, the authors examined apical precursors, isolated from E14.5 embryos and grown in conditioned media containing either WNT7A or control WNT3A. Apical precursors that were treated with WNT7A, but not those treated with WNT3A, showed a reduced frequency of spindle size asymmetry. To understand how WNT7A regulates this behavior, the authors knocked down Vangl2 using RNAi in cultured apical precursors and observed an increase in spindle size asymmetry frequency that was rescued by Vangl2 overexpression. These results suggest that spindle size asymmetry is associated with ACD and that noncanonical WNT signaling through PCP proteins maintains symmetrical spindles in dividing apical precursors.

In order to understand how WNT7A/VANGL2 signaling maintains spindle pole symmetry, the authors investigated the role of ezrin, radixin, and moesin (ERM) proteins that are associated with actin microtubules (Delaunay et al. 2014). ERM proteins act as scaffolding proteins during cell division and when phosphorylated can tether actin filaments to the cell membrane (Clucas and Valderrama 2014). Using an antibody specific to the phosphorylated versions of ERM proteins (P-ERM), a high level of P-ERM was observed during metaphase in a ringlike structure on the inner face of the plasma membrane of apical precursors. Focusing on moesin, the authors knocked down this ERM using RNAi, dissolving P-ERM immunostaining, while significantly increasing the frequency of spindle size asymmetry. Loss of Vangl2 also decreased P-ERM immunostaining, whereas treatment with WNT7A increased P-ERM immunostaining. Taken together, the data demonstrate a role for WNT7/ VANGL2/P-MOESIN in preserving spindle pole symmetry, possibly by anchoring astral microtubules to the cell cortex. 
Next, Delaunay and colleagues addressed the consequences of Vangl2 downregulation on division mode using an ex vivo clonal analysis on brain slices to track both spindle size and cell fate over time. Embryonic (E14) cortices were electroporated with plasmids encoding $G F P$ along with either Vangl2 or Vangl2 $2^{L} / L p$. The latter construct, Vangl2 $2^{L} / L p$, encodes a mutant protein lacking VANGL2 function due to a point mutation that causes mistargeting of the protein (Kibar et al. 2001). Individual daughter cells were tracked and their fate determined on the basis of their behavior and location. Expression of Vangl2 reduced the frequency of ACDs, resulting in a higher proportion of SCDs yielding two neurons. In contrast, expression of $\operatorname{Vangl} 2^{L p} / L p$ increased the proportion of cells undergoing ACD, favoring divisions that yielded an apical precursor cell and a neuron. To further explore the events occurring during these ACDs, the daughter closest to the ventricle was denoted the lower cell, whereas the basally located cell was denoted the upper cell. Under the condition of $\operatorname{Vang} 12^{L p / L p}$ overexpression, the majority of lower cells became APs, whereas upper cells became neurons. This is reverse of the results under wild-type (WT) conditions. Significant to the analysis of spindle size asymmetry, this change in identity under the mutant condition was associated with a concomitant change in the size and shape of the spindle; the smaller spindle consistently correlated with the generation of an apical precursor cell. In contrast, neurons arose from the larger spindle, suggesting that cell fate outcomes are regulated by spindle pole size.

There is a well-studied link between the mode of division and the plane of division. Indeed, the previously described paper by Le Grande and colleagues demonstrated such a role for WNT7A/FZD7/VANGL2 in specifying spindle pole orientation during the division of muscle satellite stem cells, a process that directed cell fate determination by tethering either both or only one daughter cell to the basal lamina. In this vein, Delauney and colleagues also examined the consequences of Vangl2 downregulation on the plane of division of individual apical precursors during cortical neurogenesis (Delaunay et al. 2014). Early studies on this topic indicated that during corticogenesis, divisions of cortical progenitors perpendicular to the ventricular surface usually result in SCDs, whereas horizontally shifted division planes lead to asymmetric outcomes and neurogenic differentiation (Ang et al. 2003; Chenn and McConnell 1995). Since then, however, it has become clear that a key determinant of spindle orientation is whether it results in a cleavage plane that bisects the dividing cell in ways that influence inheritance of the apical membrane that attaches the daughter to the ventricular surface (Konno et al. 2008; Kosodo et al. 2008; Noctor et al. 2008). Thus, symmetric divisions that result in both daughters inheriting apical attachments to the ventricular surface are self-renewing, whereas asymmetric divisions, even just slightly asymmetric, in which only a single daughter inherits the small apical attachment, result in differentiative divisions with one daughter adopting neuronal cell fate.

With this in mind, Delauney examined division plane orientation of apical precursors at E11.5 and E14.5 in WT Vangl2 knockdown and Vangl2 ${ }^{L p / L p}$ mice (Delaunay et al. 2014). In all genotypes, the majority of cell divisions occurred with the spindle aligned parallel to the ventricular surface and the division plane within 15 degrees of vertical, although loss of Vangl2 slightly randomized the divisions. This result indicated that spindle size asymmetry, which is regulated by WNT7A/VANGL2 signaling, regulates cell fate acquisition independent of division plane, although it is important to note that the apical attachment of 
cells was not directly monitored. Nevertheless, this was a surprising result and was further investigated by examining the relationship between spindle pole orientation/division plane and spindle size asymmetry using a dominant negative form of LGN. LGN regulates spindle positioning during asymmetric cell divisions. Loss of $L G N$ randomizes the plane of division (Konno et al. 2008), a result that was also observed by Delaunay and colleagues, who further showed that this randomization did not alter spindle size asymmetry (Delaunay et al. 2014). Taken together, these results show that spindle size asymmetry is independent of division plane because loss of Vangl2 does not substantially affect the latter while dramatically impacting the former, whereas the opposite is true for LGN.

Taken together, current studies on noncanonical WNT signaling demonstrate distinct roles in influencing cell fate determination by regulating ACD. In the muscle, WNT7A/FZD7/ VANGL2 signals to orient the plane of satellite stem cell division, fostering SCD at the expense of ACD. In contrast, during cortical neurogenesis, WNT7/VANGL2/MOESIN regulates spindle size asymmetry and governs cell fate determination independent of cell division plane or spindle orientation. These examples reveal tissue-specific deployment of signals affecting differential outcomes. From current knowledge, it seems likely that upstream activators such as different ECM components, as well as downstream effectors, such as various cytoskeletal proteins, may be the key to generating specific signaling outcomes. Additional examples of how this core noncanonical signaling pathway functions during stem cell division will be required to fully understand how WNT7/VANGL2 governs tissue morphogenesis and homeostasis.

\subsection{Hedgehog Signals Regulate Cell Fate via Oriented Cell Division}

WNTs are not the only family of extracellular cues presiding over stem cell division and cell fate acquisition during development. The Hedgehog signaling pathway also functions to control developmental programs in this manner (Alman 2015). The pathway is composed of secreted ligands, receptors, and transcription factors and exists in an "on or off" state (Fig. 16.1b). In mammals, when the pathway is off, the inhibitory receptor, Patched (PTCH), prevents the signaling receptor, Smooth-ened (SMO), from reaching the plasma membrane; instead, inactive SMO is held inside the cytoplasm within a vesicle. This results in the transcription factor GLI being processed into its repressive form by the FUSED/SUFU/KIF complex. GLI then travels to the nucleus to act as a transcriptional repressor of hedgehog target genes. When the pathway is in the "on," state, one of the Hedgehog ligands binds the PTCH receptor, which then gets degraded, and releases SMO from vesicles, allowing it to carry out signaling at the plasma membrane. SMO signaling halts the processing of GLI, leaving it in its active form. Uncleaved GLI then translocates to the nucleus where it promotes the expression of Hedgehog target genes (Fig. 16.1c). Hedgehog, well known for establishing embryonic patterning, has recently been shown to enhance proliferation in the developing cortex and cerebellum by stimulating nonterminal SCD of stem and progenitor cells. In performing this function, mounting evidence shows that SHH influences the subcellular localization of cell fate determinants by acting together with collaborating pathways such as those mediated by NOTCH (NOTC) and WNT. The ability to sequester intracellular proteins, for example, cell fate determinants to a single daughter cell, may require the precise deployment of these ligands via targeted, rather than global, delivery 
mechanisms that restrict the interaction between the extracellular cue and their cell surface receptor. This, in turn, has the potential to localize the influence of these signals to distinct regions of the receiving cell's cytoplasm.

\subsubsection{Sonic Hedgehog Signaling Regulates Cell Fate by Promoting Neuronal SCD in Radial Glial Cells During Cortical Neurogenesis}

Hedgehog signaling, specifically sonic hedgehog $(\mathrm{SHH})$, promotes cortical neurogenesis by stimulating SCD in radial glial cells (Dave et al. 2011). It has been long understood that SHH is important for cortical development (Palma and Ruiz i Altaba 2004). As described above, radial glial cells serve as neural precursors, in addition to their role as migratory scaffolds, and can either undergo one of two different modes of SCD or ACD. To expand their pool, radial glial cells divide via SCD to generate two radial glial cells. Radial glial cells, however, can also undergo a differentiative, neurogenic SCD that results in two neurons. In contrast during ACD, radial glial cells produce one neuron and one radial glial cell (Fig. 16.4a). Therefore, by regulating both the type of SCD (proliferative versus neurogenic) and the mode of division (SCD versus ACD), these cells have the capacity to regulate the number of multiple cell types in the ventricular zone. In recent studies, Dave and colleagues investigated the role of SHH signaling in this process by examining the consequences of Ptch deletion (Ptch $1^{\text {lox/lox }}$ mice), and thus activation of SHH signaling, using a clonal pair cell assay. This assay monitors the division of single radial glial cells using immunocytochemistry to track the production of either radial glial cells (GLASTpositive) or postmitotic neurons (TuJ-1-positive). An SCD yields two GLAST-positive cells (proliferative SCD) or two TuJ-1-positive cells (neurogenic SCD), whereas an ACD results in one GLAST and one TuJ-1 positive cell. The authors observed that cortical cells from $P t c 1^{l o x} / l o x$ animals, in which SHH signaling is derepressed due to the absence of this negative regulator, divided primarily via SCD, generating either two radial glial cells or two neurons (Fig. 16.4b). In contrast, WT control cells divided mainly by neurogenic SCD that promoted differentiation. In both cases, the frequency of ACD was low and remained unchanged in $W T$ control and $P t c 1^{10 x / l o x}$ cells. Thus, SHH signaling stimulates proliferative SCD of radial glial neurons at the expense of neurogenic SCD.

To further understand the link between extracellular HH signaling in regulating division mode, the authors examined Notch signaling. This pathway has been previously implicated in regulating the balance between SCD/ACD in neural stem and progenitor cells, with activation of NOTC1 and NOTC3 found to promote radial glia cell identity (Gaiano et al. 2000). Indeed, Dave and colleagues showed that activation of SHH signaling, via Ptch1 deletion, resulted in an upregulation of Notch effector proteins HES1 and BLBP, a result that connects extracellular Hedgehog with the cytoplasmic components of Notch signaling during SCD. The authors further explored the interaction between Notch and Hedgehog signaling during cortical neogenesis by deleting $R b p j$, which encodes a DNA binding protein that acts with cleaved Notch intracellular domain to regulate the transcription of Notch target genes. Performing the clonal pair assay, the authors found that loss of Rbpj in an SHH-signaling environment significantly increased the number of cells undergoing neurogenic SCD, compared to the proliferative SCD that occurs in response to SHH when 
Notch signaling is intact. These results suggest that SHH and NOTCH signaling pathways cooperate to enhance symmetric proliferative divisions of neocortical stem cells.

\subsubsection{Global Sonic Hedgehog Promotes SCD in the Developing Cerebellum: Targeted Sonic Hedgehog Promotes ACD}

In addition to promoting SCD in the cortex, recent studies demonstrate that $\mathrm{SHH}$ also stimulates this mode of division to expand the population of granule neuron progenitors (GNPs) in the cerebellum. There are multiple proliferative zones in the embryonic and early postnatal brain; one is the ventricular zone (discussed in the previous section) and another is the external granule layer (EGL) of the cerebellum. Granule neurons are a large class of neurons with a unique pattern of differentiation, occurring in two migratory phases that are divided by a proliferative phase. During the first phase, GNPs arise from the rhombic lip located next to the ventricular zone and migrate to the surface of the cerebellum, forming the EGL (Miale and Sidman 1961). In this layer, GNPs are highly proliferative. The second phase of migration begins when the GNPs become postmitotic and migrate from the EGL inwards on Bergmann glial fibers through the Purkinje cell layer to form mature granule neurons of the internal granule layer (IGL) (Komuro et al. 2001). Studies have shown that a variety of factors affect the thickness of the EGL, including SHH, which increases EGL thickness by fostering GNP proliferation (Wechsler-Reya and Scott 1999). GNPs, like muscle satellite and other types of stem cells, divide into two orientations with respect to the pial or outer surface of the cerebellum: parallel or perpendicular. Yet, it is unclear whether factors like SHH, which stimulate GNP proliferation, also regulate spindle pole orientation and cell fate determination.

Increasingly clear, however, is the fact that networks of local signals, both soluble and membrane-bound niche factors, regulate these stem cell divisions. It is no surprise, then, that SHH and WNT/ $\beta$-catenin signaling pathways have emerged as two such intertwined pathways governing spindle pole orientation and cell fate determination (Haldipur et al. 2015). In their recent paper, Haldipur and colleagues used immunohistochemistry with antibodies against phosphohistone- 3 to examine the division plane of mitotic GNPs, from which spindle orientation can be determined. The authors found that between postnatal days 0 and 4, half of GNPs divided with spindle poles parallel and half with spindle poles perpendicular to the pial surface. The number of cell division with spindles perpendicular to the pial surface rose gradually between postnatal days 5 and 14 (Fig. 16.5a). Next, the authors investigated the effect of SHH on division orientation by treating pups with cyclopamine, a drug that inhibits SHH signaling, or an agonist of smoothened (SAG), which stimulates the pathway. The EGL of cyclopamine treated animals was thinner compared to control and a significantly higher percentage of cells divided with spindles perpendicular to the pial surface, corresponding to an increase in cells expressing the differentiation marker NeuroD1. In contrast, SAG-treated animals displayed a concordant increase in the percentage of parallel spindles and a reduced number of NeuroD1-positive cells. Thus, SHH appears to favor symmetric GNP divisions in which both cells are aligned along the outer pial surface and retain their progenitor status. This is the same SHH effect observed in the cortex (Dave et al. 2011) and similar to the effect of noncanonical WNT7A signaling on 
muscle satellite stem cells in which aligned cell divisions maintain stem cell contact to the outer tissue surface, resulting in population expansion via SCD.

In order to elucidate a possible mechanism for the influence of SHH on GNP oriented division, the authors first probed for $\beta$-catenin in anaphase cells of the EGL (Haldipur et al. 2015). They observed an asymmetric cellular distribution of $\beta$-catenin but no correlation between this asymmetric distribution and the plane of cell division. A recent study in murine embryonic stem cells, however, has showed that an asymmetric distribution of $\beta$-catenin can be produced by contact of the cell with a point source of WNT3A (Habib et al. 2013). To investigate whether GNPs are similarly influenced by focal contact with WNT3A or SHH, the authors seeded GNPs at low density on coverslips that were printed with stripes of either SHH or WNT3A and immunostained for $\beta$-catenin. Paired cells, in which one cell was in contact with the stripe and the other was outside the striped region, were examined for the intracellular distribution of $\beta$-catenin. Similar to the previous results (Habib et al. 2013), the authors found that $\beta$-catenin preferentially and asymmetrically localized to the cell that was in contact with WNT3A stripe. In contrast, when both cell nuclei were in contact with the stripe, $\beta$-catenin was symmetrically distributed in both daughters. SHH stripes produced similar, but not as dramatic, results. Taken together, these experiments indicate that $\mathrm{SHH}$, locally and asymmetrically presented, may intersect with WNT signaling and have the capacity to regulate the subcellular localization of $\beta$-catenin and generate ACDs. However, as observed in the developing cortex, global stimulation by SHH enhances expansion of GNPs via SCD.

\subsubsection{Sonic Hedgehog Signaling Determines Granule Neuron Progenitor Fate by Maintaining the Balance Between SCD and ACD in the Cerebellum}

The studies by Haldipur and colleagues demonstrated the importance of SHH in favoring GNP expansion via SCD and showed how reduced or focal delivery of SHH shifted the division balance toward differentiation. However, these experiments did not track the acquisition of cell fate by individual GNPs as they proliferate in response to SHH. This investigation was recently tackled by Yang and colleagues using mice that express dual reporters: progenitor specific (Math1-GFP) and neuronal specific (Dcx-DsRed) (Yang et al. 2015). In this model, daughter cells that fluoresce the same color were the product of an SCD as evidenced by the production of two cells expressing the same fate determinant, whereas daughter cells of different colors were generated via ACD and have different cell fates (Fig. 16.5). in order to explore the role of SHH signaling on GNP fate acquisition, the authors first established a baseline by quantifying the $W T$ frequency of SCD/ACD. The authors visualized GNPs, both in dissociated culture and in freshly dissected whole-mounted cerebella using time-lapsed imaging. This analysis defined three different cell fate outcomes produced by two division modes. There were two types of SCD; one produced two progenitor cells (both cells positive for Math1-GFP) and was therefore considered nonterminal. The second type of SCD was considered terminal because it produced two intermediate cells (both positive for both Math1-GFP and Dcx-DsRed) that subsequently differentiate into granule cells. In addition, ACD was observed, producing one terminal intermediate cell (Math1-GFP/Dcx-DsRed positive) and one nonterminal progenitor (only Math1-GFP positive) (Fig. 16.5). At P4, corresponding to an early stage of neurogenesis, the 
vast majority of divisions were SCD and expansive, producing two progenitor cells. However, at a later stage (P10), the balance shifted to terminal SCD, producing two intermediate cells that differentiated into granule cells. A minority $(<5 \%)$ of cells underwent $\mathrm{ACD}$, renewing the progenitor cell while also forming an intermediate cell that will differentiate into a granule cell. Taken together, these data show that GNPs divide primarily by SCD and differentiation to granule cells occurs through an intermediate cell that expresses both progenitor and neuronal markers.

The authors used two methods to stimulate SHH signaling in the dual reporter mice (Math1GFP;Dcx-DsRed) (Yang et al. 2015). They either activated SHH signaling in culture using recombinant SHH (C25II) or they crossed the reporter mice to a Ptch1 ${ }^{-1-}$ line in which SHH signaling is enhanced. In response to SHH activation, the number of Math-GFP progenitors increased in proportion to the other cell types (intermediate and differentiated cells) over the time course of neurogenesis, suggesting an increase in progenitor SCD that delays cerebellar neurogenesis. Blockade of the SHH pathway, by either adding cyclopamine to GNP cultures or injecting it into Math1-GFP;Dcx-DsRed;Ptch ${ }^{+-}$mice, reversed the expansive division mode, increasing both $\mathrm{ACD}$ and the terminal type of SCD that produces granule cells, at the expense of expansive, nonterminal SCDs that produce progenitor cells. Analysis of division planes by phosphohistone- 3 immunostaining in $W T$ versus $P t c h 1^{+/-}$mice revealed a decrease in parallel divisions (i.e., with spindle poles perpendicular to the pial surface). This result is consistent with the increase in SCD observed by these authors and by Halipur and colleagues who stimulated SHH signaling using SAG treatment. Together, the data demonstrate that SHH enhances proliferation by regulating both division mode (ACD/SCD) and type (nonterminal/terminal).

These examples of SHH activity in the cortex and cerebellum show how widespread SHH stimulation expands stem and progenitor populations by favoring SCD. However, the mechanism by which SHH controls the ratio of cells undergoing SCD versus ACD and how it contributes to cell fate acquisition is still mysterious. Conceivably, division mode and type may be regulated by the concentration and localization of SHH in the proliferative zone of the neocortex and in the EGL of the cerebellum (Komada et al. 2008; Martinez et al. 2013; Wallace 1999). During secretion, SHH is modified by palmitoylation as well as by a cholesterol modification, which occurs during an autoprocessing cleavage event. These modifications ensure that the extracellular movement of SHH is highly regulated as it is trafficked to the plasma membrane, stabilized on the cell surface, transferred to carrier lipoproteins, and released from the cell in large soluble lipoprotein complexes or spread by filopodia-like extensions (Briscoe and Therond 2013). This raises the possibility that Hedgehog, rather than globally bathing GNPs, is delivered in a highly regulated manner that governs division mode and type. In this model, regulated release would be developmentally controlled to provide for ample SHH distribution during GNP expansion via SCD at early time periods. In contrast, highly localized delivery at later stages of development would specify differentiation. These events may occur in collaboration with WNT and NOTCH signaling to further refine the ways stem and progenitor cells divide and acquire cell fates by regulating the subcellular localization of fate determinants. 


\subsection{Axon Guidance Molecules Driving ACD Through the Spindle}

Neural development is complex and numerous secreted factors are likely to regulate the proliferation and cell fate acquisition of neurons as well as their migration to new environments. One type of signaling involves cues that were originally identified as axon guidance molecules, directing neurons and their growth cones to proper targets in the embryo. These guidance cues govern migration by regulating cytoskeletal dynamics, but more recently they have a newly respected role in regulating cell proliferation, including ACD, not only during neural development (Borrell et al. 2012) but also in epithelial organs such as the breast (Ballard et al. 2015).

SLITs are secreted ligands that bind to ROBO receptors to regulate multiple developmental programs. There are three SLIT ligands (Slits 1-3) and four ROBO receptors (Robo 1-4). Activation of the SLIT/ROBO pathway leads to a cascade of signaling events that range from axon guidance to cell proliferation (Ballard and Hinck 2012). Recently, SLIT/ROBO signaling has been implicated in the maintenance of mammary stem cells. The mammary gland is a dynamic organ that undergoes many rounds of growth and expansion with each estrus cycle and pregnancy (Macias and Hinck 2012). At the onset of puberty, the mammary gland grows from a rudimentary ductal structure to a fully arborized tree over a span of 6 weeks. Fueling this growth are the highly proliferative cells in the terminal end buds that generate ductal cells as they push through the fat pad, directing growth toward the unfilled space (Fig. 16.6a). Once the terminal end buds reach the outer edge of the fat pad, they dissolve leaving behind a bilayered ductal structure containing a heterogeneous luminal population and a basal layer, which contains stem cells. To fuel proliferative cycles, the mammary gland maintains a reserve of stem cells into adulthood. The inside of a terminal end bud serves as the hub for stem and progenitor cells during development and is made of inner body cells and an outer layer of cap cells (Fig. 16.6b). It is in the terminal end buds where the vast majority of ACD occurs (Ballard et al. 2015). Mammary stem cells utilize $\mathrm{ACD}$ to both self-renew and differentiate into the various cell types that compose the mammary duct. Stem cell self-renewal is fundamental to the function of the mammary gland, and recent evidence shows that SLIT/ROBO signaling plays a role in regulating this process.

In a study by Ballard and colleagues, SLIT2/ROBO1 signaling was shown to regulate the expression of Inscuteable (INSC), which in turn governs the balance between ACD and SCD (Ballard et al. 2015). INSC is a central component of the spindle orientation complex and is initially recruited by the PAR complex and engages with LGN. Acting as a molecular baton, INSC hands off LGN to NuMA, resulting in the asymmetric co-localization of LGN and NuMA at the apical pole (Mapelli and Gonzalez 2012). This LGN/NuMA complex facilitates spindle pole tethering, thereby contributing to the unequal distribution of cell fate determinants. The authors first showed that loss of Robol increases Insc levels, with no change in the expression of $L g n$ or $N u M A$. SLIT/ROBO signaling regulates Insc expression by governing the subcellular localization of the transcription factor Snail (SNAI1) through the PI3kinase/AKT/GSK-3 $\beta$ pathway. Loss of Robo1 sends SNAI1 to the nucleus where it directly enhances Insc transcription. 
Ballard and colleagues used two assays to demonstrate how excess INSC, generated by either loss of Robol (Robol ${ }^{-1}$ ) or transgenic overexpression of Insc (Insc ${ }^{\mathrm{KI} / K I}$ ), decreases the frequency of ACDs in mammary end buds (Ballard et al. 2015). In the first assay, the stem cell containing basal population of mammary cells was isolated by fluorescence activated cell sorting (FACS), labeled with a membrane permeable dye, PKH26, and plated at single cell density in Matrigel. PKH26 binds to cell membranes and is distributed to daughter cells upon division. ACDs generate a quiescent daughter stem cell, which maintains its fluorescence, and a progenitor cell that continues to divide and dilute the dye, resulting in a colony with a single labeled cell. In contrast, SCDs dilute the dye with each division, resulting in unlabeled colonies. Excess INSC resulted in more unlabeled colonies, indicating fewer ACDs. In the second assay, immunohistochemistry was used to visualize the co-localization of NuMA and LGN in crescent-like structures asymmetrically located over one spindle pole in mammary end bud cells. Excess INSC resulted in fewer of these crescent-like structures, again suggesting that fewer ACDs occurred in $\mathrm{Robol}^{-1}$ and Insc ${ }^{K I / K I}$ tissue.

The consequences of excess INSC expression on the mammary gland were investigated by serial passaging and limiting dilution assays that measure the robustness and number of mammary stem cells (Ballard et al. 2015). Mammary stem cells were enriched using FACS to isolate basal cells. For serial passaging, cells were plated at single cell density in Matrigel and passaged every 7 days. Cells expressing elevated levels of Insc generated larger colonies that passage longer compared to $W T$ cells. For limiting dilution assays, a serially reduced number of enriched basal cells were transplanted into precleared mammary fat pads. After 8 weeks, the frequency and size of mammary outgrowths were measured, allowing an estimation of mammary stem cell number. Cells containing excess Insc, harvested from either Robo1 $^{-1}$ or Insc ${ }^{K I / K I}$ mammary tissue, contained threefold more mammary stem cells compared to $W T$ tissue. Together, these experiments demonstrate that SLIT/ROBO/SNAI1 signaling through INSC promotes ACD, with the loss of Robol shifting the balance toward SCD due to upregulated Insc expression, which interferes with correct spindle pole positioning (Fig. 16.6c).

With this example, a number of themes come into focus in vertebrate tissue. Increasingly, evidence suggests that extracellular cues send signals to the mitotic spindle to regulate its orientation and size. These changes in the mitotic spindle govern the asymmetric distribution of cell fate determinants. Focal, rather than global, extrinsic signaling may be required to regulate spindle orientation and generate disproportionate signaling in daughter cells. For example, in the satellite stem cell niche, rotation of the spindle places one daughter in contact with the sarcolemma and the other in contact with the basal lamina of the muscle fiber. One explanation is that each location provides a distinct niche characterized by a different set of extracellular factors. Not all stem cell niches, however, are as geometrically constrained and allow for such discrete daughter cell interactions. This means that elaborate, and still largely unknown, mechanisms are likely required to regulate the extracellular distributions of extrinsic factors. Like WNT and Hedgehog proteins, the extracellular availability of SLIT is regulated by a number of extracellular matrix components, for example, heparin sulfate proteo-glycans (Ballard and Hinck 2012). Such control has the capacity to create discrete niches and govern the delivery and differential activation of these 
ligands in temporally and spatially restricted manners. Future studies to improve our understanding of the extracellular environments governing the availability of signaling molecules that function in controlling stem cell division will further our knowledge of stem cell population dynamics in vertebrate tissues.

\subsection{Conclusion: Implications of Extracellular Cues that Govern the Mode of Stem Cell Division in Medicine}

In this chapter, we reviewed current research that describes the extracellular cues-WNT, SHH, and SLIT - and how they regulate cell division mode in the muscle, brain, and breast. Due to their critical role in regulating stem cell renewal and homeostasis, these extracellular cues and morphogens may be specific therapeutic targets for the development of treatments for diseases characterized by aberrant cell division mode. For example, WNT7A promotes expansion of the stem cell pool and therefore should be explored for developing novel therapies to combat degenerative diseases. In contrast, the loss of Slit2 drives tumor proliferation, making SLIT2 pathway components potential therapeutic targets for translational efforts to fight cancer.

\subsubsection{WNT7A as a Useful Agent in Duchenne Muscular Dystrophy}

The studies performed by Le Grand, Delauney, and colleagues demonstrate the role of WNT7A signaling in regulating ACD through the PCP pathway. Specifically, Le Grand showed that WNT7A drives the expansion of satellite stem cells in skeletal muscle, thereby demonstrating its property as a growth factor. There is now emerging evidence for WNT7A in treating Duchenne Muscular Dystrophy (DMD), a genetic childhood disorder that results in progressive muscle weakness leading to death by age 30. In a recent study by Maltzahn and colleagues, WNT7A was used as a treatment agent in a mouse model of DMD (von Maltzahn et al. 2012). Animals treated with WNT7A increased their satellite stem cell number, which subsequently resulted in increased muscle strength and reduced damage in response to injury. These positive results make WNT7A a prime candidate for DMD therapeutics.

\subsubsection{SLIT2 as a Therapeutic Breast Cancer Agent}

Another molecule with promising therapeutic uses is SLIT2 in the treatment of breast cancer. In the study done by Ballard and colleagues, SLIT2 signaling through ROBO1 maintained the balance between ACD and SCD. Loss of SLIT2 signaling resulted in both an increase in SCD (Ballard et al. 2015) and the formation of hyperplastic lesions in normal breast tissue (Marlow et al. 2008). Taken together, these data suggest that loss of Slit2, which occurs in $40 \%$ percent of basal breast tumors (Cancer Genome Atlas 2012), may lead to increased proliferation due to the symmetric expansion of cancer stem/progenitor cells. To this end, a recent review by $\mathrm{Gu}$ and colleagues outlines multiple studies that use SLIT2 in cultured breast cancer cells or mouse tumor models to prevent the proliferation and metastasis of breast tumors (Gu et al. 2015). 


\subsubsection{SHH as a Target in Medulloblastoma}

SHH, like SLIT, has signaling properties that can be exploited in cancer treatment. Overactivation of SHH signaling in the cerebellum leads to expansion of progenitor cells and cancers such as medulloblastoma (Kieran 2014; Yang et al. 2015); therefore, drugs inhibiting SHH signaling have garnered a great deal of attention and may represent promising therapeutic agents. Medulloblastoma is a pediatric cancer that affects the cerebellum and presents as four distinct subtypes. SHH overexpression is a marker for one subtype characterized by an intermediate prognosis. Recent efforts in drug design have been aimed at developing inhibitors that block SHH signaling in this subtype. Robinson and colleagues show that treatment of patients with the SHH inhibitor, Vismodegib, prevented cancer recurrence in a phase II clinical trial (Robinson et al. 2015). These results demonstrate that silencing the SHH pathway may halt cancer progression.

In conclusion, SHH, WNT, and SLIT are extracellular cues that have important developmental roles in multiple tissues and represent promising targets in drug discovery. Furthermore, these molecules are examples of how understanding the function of proteins during development leads to progress in combating disease.

\section{Acknowledgments}

This work was supported by the NIH: GMI-098897 R01 to LH and HGRI-R25HG006836 predoctoral support to PS.

\section{References}

Alman BA. The role of hedgehog signalling in skeletal health and disease. Nat Rev Rheumatol. 2015; 11(9):552-560. [PubMed: 26077918]

Ang ES Jr, Haydar TF, Gluncic V, Rakic P. Four-dimensional migratory coordinates of GABAergic interneurons in the developing mouse cortex. J Neurosci. 2003; 23(13):5805-5815. [PubMed: 12843285]

Ballard MS, Hinck L. A roundabout way to cancer. Adv Cancer Res. 2012; 114:187-235. [PubMed: 22588058]

Ballard MS, Zhu A, Iwai N, Stensrud M, Mapps A, Postiglione MP, Knoblich JA, Hinck L. Mammary stem cell self-renewal is regulated by Slit2/Robo1 signaling through SNAI1 and mINSC. Cell Rep. 2015; 13(2):290-301. [PubMed: 26440891]

Borrell V, Cardenas A, Ciceri G, Galceran J, Flames N, Pla R, Nóbrega-Pereira S, García-Frigola C, Peregrín S, Zhao Z, Ma L, Tessier-Lavigne M, Marin O. Slit/Robo signaling modulates the proliferation of central nervous system progenitors. Neuron. 2012; 76(2):338-352. [PubMed: 23083737]

Briscoe J, Therond PP. The mechanisms of Hedgehog signalling and its roles in development and disease. Nat Rev Mol Cell Biol. 2013; 14(7):416-429. [PubMed: 23719536]

Cancer Genome Atlas Network. Comprehensive molecular portraits of human breast tumours. Nature. 2012; 490(7418):61-70. [PubMed: 23000897]

Ceron J, Tejedor FJ, Moya F. A primary cell culture of Drosophila postembryonic larval neuroblasts to study cell cycle and asymmetric division. Eur J Cell Biol. 2006; 85(6):567-575. [PubMed: 16621131]

Chenn A, McConnell SK. Cleavage orientation and the asymmetric inheritance of Notch1 immunoreactivity in mammalian neurogenesis. Cell. 1995; 82(4):631-641. [PubMed: 7664342]

Clucas J, Valderrama F. ERM proteins in cancer progression. J Cell Sci. 2014; 127(Pt 2):267-275. [PubMed: 24421310] 
Dave RK, Ellis T, Toumpas MC, Robson JP, Julian E, Adolphe C, Bartlett PF, Cooper HM, Reynolds BA, Wainwright BJ. Sonic hedgehog and notch signaling can cooperate to regulate neurogenic divisions of neocortical progenitors. PLoS One. 2011; 6(2):e14680. [PubMed: 21379383]

De A. Wnt/Ca ${ }^{2+}$ signaling pathway: a brief overview. Acta Biochim Biophys Sin (Shanghai). 2011; 43(10):745-756. [PubMed: 21903638]

Delaunay D, Cortay V, Patti D, Knoblauch K, Dehay C. Mitotic spindle asymmetry: a Wnt/PCPregulated mechanism generating asymmetrical division in cortical precursors. Cell Rep. 2014; 6(2):400-414. [PubMed: 24412369]

Devenport D. The cell biology of planar cell polarity. J Cell Biol. 2014; 207(2):171-179. [PubMed: 25349257]

Dumont NA, Wang YX, Rudnicki MA. Intrinsic and extrinsic mechanisms regulating satellite cell function. Development. 2015; 142(9):1572-1581. [PubMed: 25922523]

Gaiano N, Nye JS, Fishell G. Radial glial identity is promoted by Notch1 signaling in the murine forebrain. Neuron. 2000; 26(2):395-404. [PubMed: 10839358]

Gauthier-Fisher A, Lin DC, Greeve M, Kaplan DR, Rottapel R, Miller FD. Lfc and Tctex-1 regulate the genesis of neurons from cortical precursor cells. Nat Neurosci. 2009; 12(6):735-744. [PubMed: 19448628]

Gu F, Ma Y, Zhang J, Qin F, Fu L. Function of Slit/Robo signaling in breast cancer. Front Med. 2015; 9(4):431-436. [PubMed: 26542734]

Habib SJ, Chen BC, Tsai FC, Anastassiadis K, Meyer T, Betzig E, Nusse R. A localized Wnt signal orients asymmetric stem cell division in vitro. Science. 2013; 339(6126):1445-1448. [PubMed: 23520113]

Haldipur P, Sivaprakasam I, Periasamy V, Govindan S, Mani S. Asymmetric cell division of granule neuron progenitors in the external granule layer of the mouse cerebellum. Biol Open. 2015; 4(7): 865-872. [PubMed: 25979710]

Kibar Z, Vogan KJ, Groulx N, Justice MJ, Underhill DA, Gros P. Ltap, a mammalian homolog of Drosophila Strabismus/Van Gogh, is altered in the mouse neural tube mutant Loop-tail. Nat Genet. 2001; 28(3):251-255. [PubMed: 11431695]

Kieran MW. Targeted treatment for sonic hedgehog-dependent medulloblastoma. Neuro Oncol. 2014; 16(8):1037-1047. DOI: 10.1093/neuonc/nou109 [PubMed: 24951114]

Komada M, Saitsu H, Kinboshi M, Miura T, Shiota K, Ishibashi M. Hedgehog signaling is involved in development of the neocortex. Development. 2008; 135(16):2717-2727. [PubMed: 18614579]

Komuro H, Yacubova E, Yacubova E, Rakic P. Mode and tempo of tangential cell migration in the cerebellar external granular layer. J Neurosci. 2001; 21(2):527-540. [PubMed: 11160432]

Konno D, Shioi G, Shitamukai A, Mori A, Kiyonari H, Miyata T, Matsuzaki F. Neuroepithelial progenitors undergo LGN-dependent planar divisions to maintain self-renewability during mammalian neurogenesis. Nat Cell Biol. 2008; 10(1):93-101. [PubMed: 18084280]

Kosodo Y, Toida K, Dubreuil V, Alexandre P, Schenk J, Kiyokage E, et al. Cytokinesis of neuroepithelial cells can divide their basal process before anaphase. EMBO J. 2008; 27(23):31513163. [PubMed: 18971946]

Le Grand F, Jones AE, Seale V, Scime A, Rudnicki MA. Wnt7a activates the planar cell polarity pathway to drive the symmetric expansion of satellite stem cells. Cell Stem Cell. 2009; 4(6):535547. [PubMed: 19497282]

Luer K, Technau GM. Single cell cultures of Drosophila neuroectodermal and mesectodermal central nervous system progenitors reveal different degrees of developmental autonomy. Neural Dev. 2009; 4:30. [PubMed: 19650920]

Macias H, Hinck L. Mammary gland development. Wiley Interdiscip Rev Dev Biol. 2012; 1(4):533557. [PubMed: 22844349]

Mapelli M, Gonzalez C. On the inscrutable role of Inscuteable: structural basis and functional implications for the competitive binding of NuMA and Inscuteable to LGN. Open Biol. 2012; 2(8):120102. [PubMed: 22977735]

Marlow R, Strickland P, Lee JS, Wu X, Pebenito M, Binnewies M, et al. SLITs suppress tumor growth in vivo by silencing Sdf1/Cxcr4 within breast epithelium. Cancer Res. 2008; 68(19):7819-7827. [PubMed: 18829537] 
Martinez C, Cornejo VH, Lois P, Ellis T, Solis NP, Wainwright BJ, Palma V. Proliferation of murine midbrain neural stem cells depends upon an endogenous sonic hedgehog (Shh) source. PLoS One. 2013; 8(6):e65818. [PubMed: 23776550]

Miale IL, Sidman RL. An autoradiographic analysis of histogenesis in the mouse cerebellum. Exp Neurol. 1961; 4:277-296. [PubMed: 14473282]

Morin X, Bellaiche Y. Mitotic spindle orientation in asymmetric and symmetric cell divisions during animal development. Dev Cell. 2011; 21(1):102-119. [PubMed: 21763612]

Noctor SC, Martinez-Cerdeno V, Kriegstein AR. Distinct behaviors of neural stem and progenitor cells underlie cortical neurogenesis. J Comp Neurol. 2008; 508(1):28-44. [PubMed: 18288691]

Palma V, Ruiz i Altaba A. Hedgehog-GLI signaling regulates the behavior of cells with stem cell properties in the developing neocortex. Development. 2004; 131(2):337-345. [PubMed: 14681189]

Polesskaya A, Seale P, Rudnicki MA. Wnt signaling induces the myogenic specification of resident CD45+ adult stem cells during muscle regeneration. Cell. 2003; 113(7):841-852. [PubMed: 12837243]

Robinson GW, Orr BA, Wu G, Gururangan S, Lin T, Qaddoumi I, Packer RJ, Goldman S, Prados MD, Desjardins A, Chintagumpala M, Takebe N, Kaste SC, Rusch M, Allen SJ, Onar-Thomas A, Stewart CF, Fouladi M, Boyett JM, Gilbertson RJ, Curran T, Ellison DW, Gajjar A. Vismodegib exerts targeted efficacy against recurrent sonic hedgehog-subgroup medulloblastoma: results from phase II pediatric brain tumor consortium studies PBTC-025B and PBTC-032. J Clin Oncol. 2015; 33(24):2646-2654. [PubMed: 26169613]

von Maltzahn J, Renaud JM, Parise G, Rudnicki MA. Wnt7a treatment ameliorates muscular dystrophy. Proc Natl Acad Sci USA. 2012; 109(50):20614-20619. [PubMed: 23185011]

Wallace VA. Purkinje-cell-derived Sonic hedgehog regulates granule neuron precursor cell proliferation in the developing mouse cerebellum. Curr Biol. 1999; 9(8):445-448. [PubMed: 10226030]

Wechsler-Reya RJ, Scott MP. Control of neuronal precursor proliferation in the cerebellum by Sonic Hedgehog. Neuron. 1999; 22(1):103-114. [PubMed: 10027293]

Yang R, Wang M, Wang J, Huang X, Yang R, Gao WQ. Cell division mode change mediates the regulation of cerebellar granule neurogenesis controlled by the sonic hedgehog signaling. Stem Cell Rep. 2015; 5(5):816-828. 


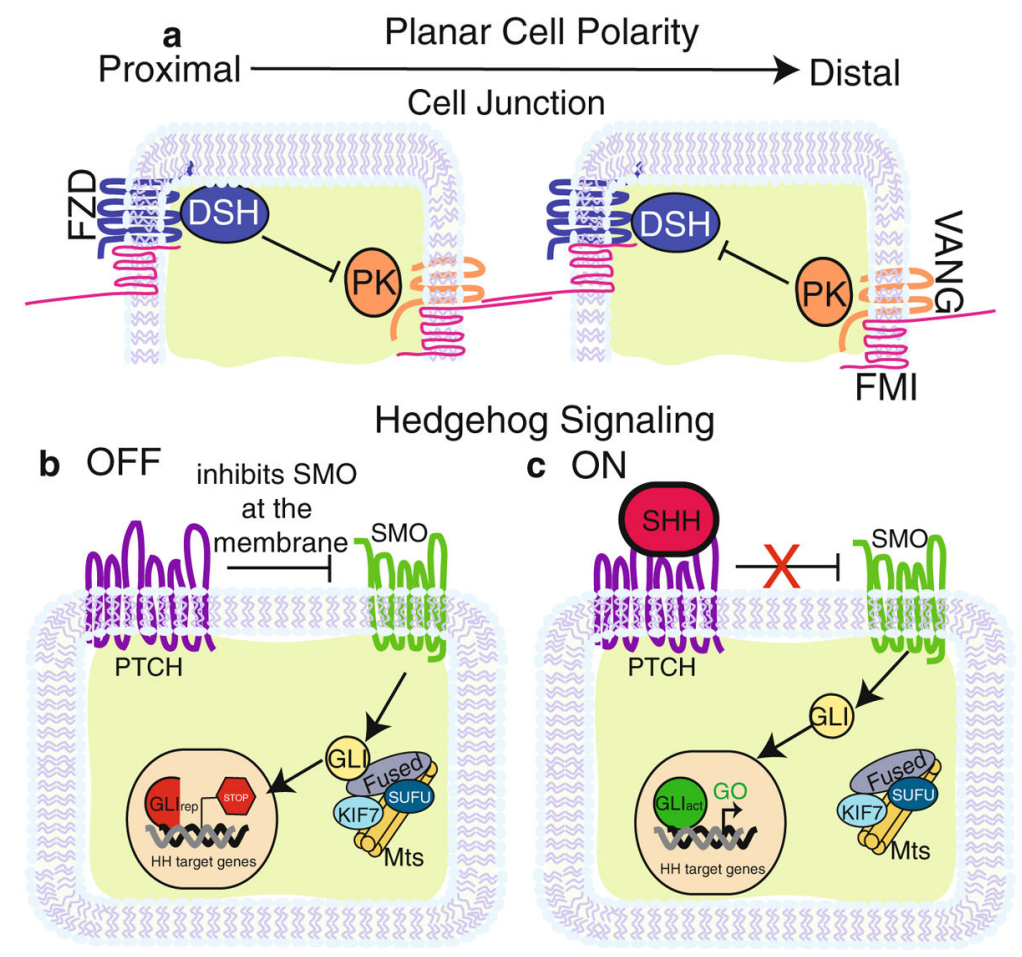

Fig. 16.1.

PCP and SHH Signaling pathways. (a) Core planar cell polarity proteins asymmetrically distributed in two epithelial cells. Epithelial cells in contact with each other partition one group of proteins (FZD, DSH shown in blue) to the proximal side of the cell and another group (VANG, PK shown in orange) to the distal. FMI (shown in pink) forms a complex with FZD at the proximal surface of the membrane and VANG on the distal. Across cell junctions, FMI from one cell binds to FMI on the adjacent cell, which connects each cell within a sheet of epithelium. DSH and PK maintain asymmetry in a cell by functioning as inhibitors of each other. (b) In the absence of SHH, the pathway is in an "off" state in which PTCH (purple) inhibits SMO (green) at the cell membrane. SMO is then held captive on the membrane of vesicles (not depicted). This activates a complex of FUSED, SUFU, KIF7, and microtubules to process GLI into its transcriptional repressive form. The result is the repression of Hedgehog target genes. (c) In the "on" state, Sonic Hedgehog (pink) binds to PTCH, which relieves the repression on SMO. After SMO activation, GLI is left unprocessed and is able to enter the nucleus to stimulate Hedgehog target gene expression 
a

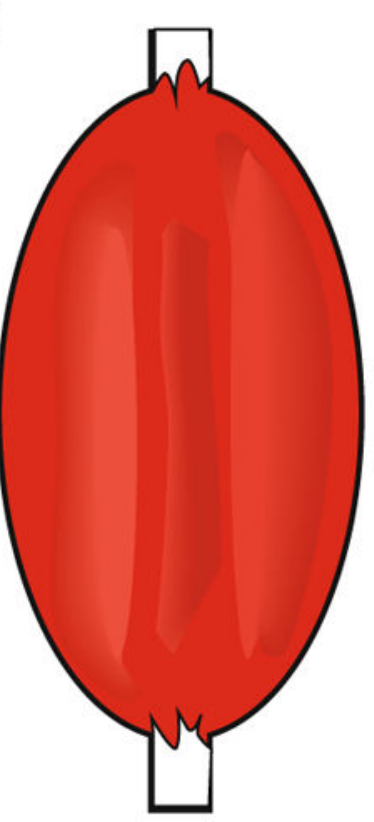

b Satellite

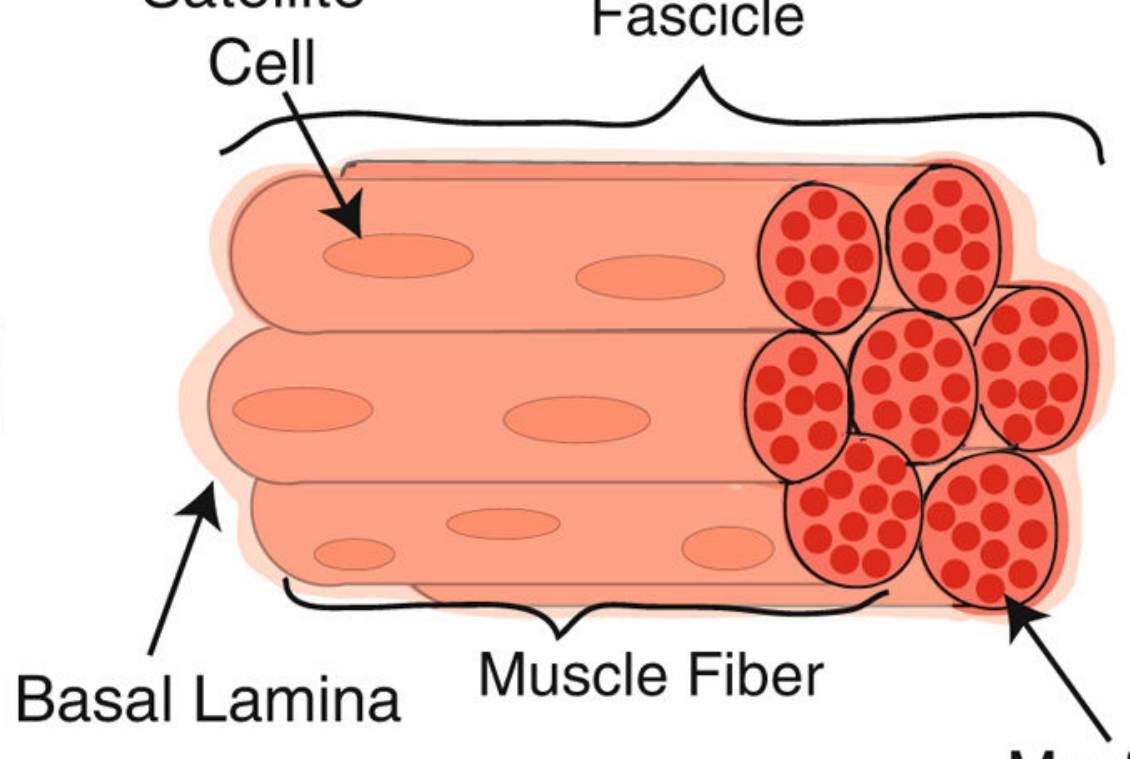

C

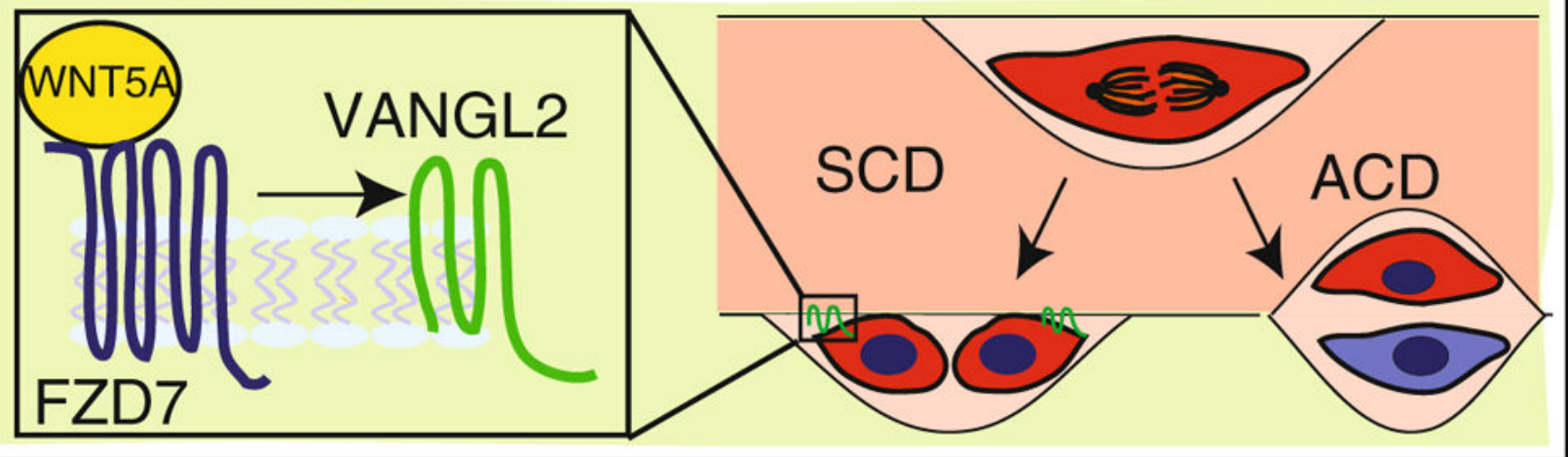

Fig. 16.2.

WNT/PCP signals during SCD in satellite stem cells. (a) Cartoon of a limb comprising skeletal muscle and bone. (b) Skeletal muscle composed of seven fascicles, each of which contains muscle fibers (seven depicted here). Within each muscle fiber are multiple myofibrils. The satellite stem cells (red) reside in between the encapsulating basal lamina and the sarcolemma (not shown) of each muscle fiber. (c) Satellite stem cells undergo both SCD and ACD. The left arrow indicates the outcome of an SCD where both daughter cells are in contact with the sarcolemma anchored by VANGL2 (green). The right arrow indicates an ACD in which only one daughter cell is in contact with the sarcolemma, remaining a satellite stem cell (red), and the other daughter contacts the basal lamina, becoming a progenitor cell (blue). The enlarged inset depicts WNT5A (yellow) bound to FZD7 (blue) during an SCD, triggering the accumulation of VANGL2 on opposite poles of the daughter cells 
a

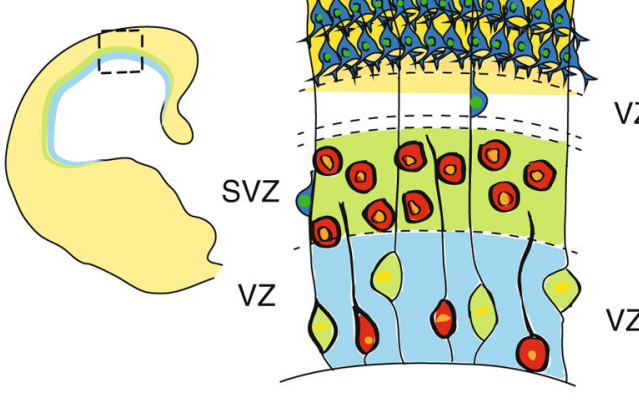

c

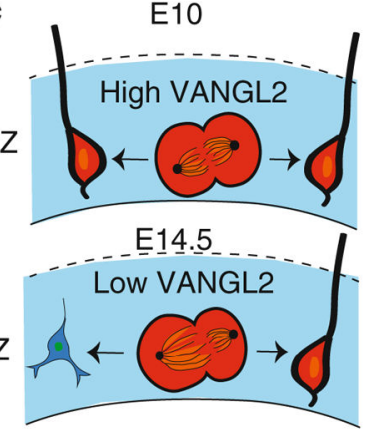

Fig. 16.3.

Spindle size asymmetry and PCP in the cortex. (a) Cartoon of a section through a neonatal cortex. The ventricular zone (VZ) is in blue, the subventricular zone (SVZ) is in green, and the mature cortical layer is in yellow. (b) Enlarged view of the boxed area in (a) showing that each layer is composed of different cell types. Residing in the VZ are the apical precursors (red) and glial cells (green). The apical precursors remain quiescent or undergo cell division while the glial cells serve as a migratory scaffold for neurons moving up to the mature cortical layer. The SVZ contains precursor cells (red). The cortical plate (CP) in yellow houses mature neurons. (c) In the VZ, the level of VANGL2 expression determines ACD versus SCD. At embryonic day 10.5, high VANGL2 in dividing apical precursors leads to spindles with equal size resulting in an SCD. This generates two apical precursor cells. By embryonic day 14.5, VANGL2 expression decreases, leading to spindle size asymmetry and an increase in ACDs. This produces a precursor and a neuron 


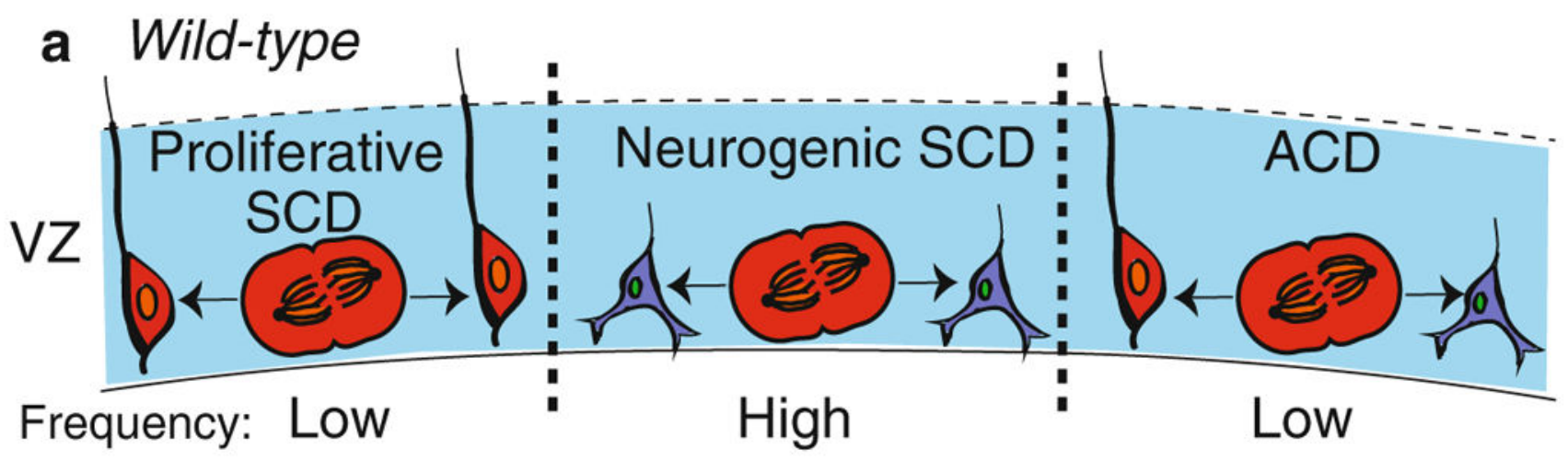

\section{b SHH activation ( Ptch -/-)}

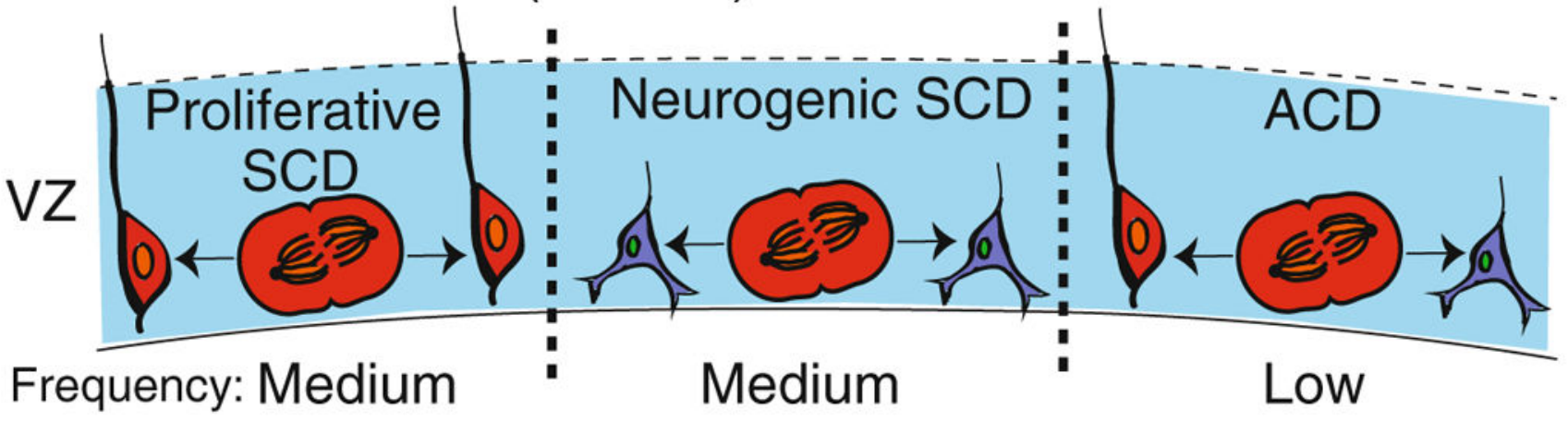

Fig. 16.4.

SHH and cell division in the VZ of a neocortex. (a) In a wild-type brain, radial glial cells (red) divide via proliferative SCD (left) to generate two radial glial cells, neurogenic SCD (middle) to create two neurons (blue) or ACD (right) to produce a radial glial cell and a neuron. (b) Upon SHH activation in a $\mathrm{Ptch}^{-/}$mutant, radial glial cells increase the frequency of proliferative SCD. The frequency of each division type is indicated 


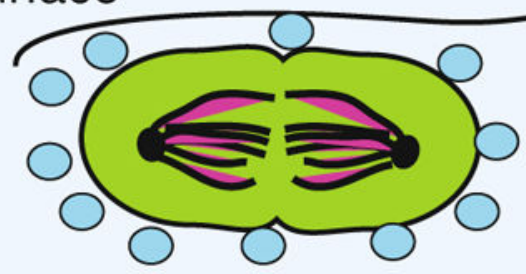

Non-Terminal SCD

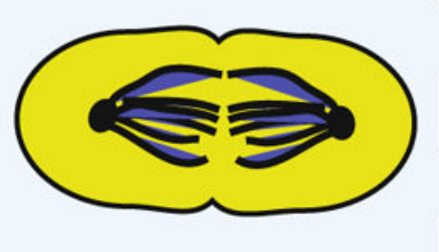

Terminal SCD

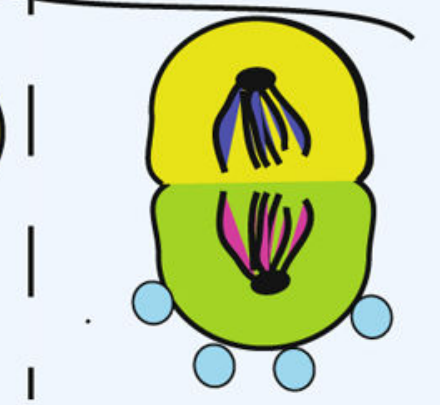

Fig. 16.5.

Model for regulation of cell division and fate specification by SHH in the cerebellum. In the cerebellum, GNPs can divide so that their spindles are parallel to the pial surface (left of dashed line) or perpendicular to the pial surface (right). (a) During a parallel division, both daughter cells are exposed to the same level of SHH (blue circles) and retain their GNP fate, resulting in a nonterminal SCD (green cells). In the absence of SHH, both daughter cells undergo a fate change, causing terminal SCD (yellow cells). (b) During a perpendicular division, SHH is exposed locally to one daughter cell and not the other, resulting in an ACD that produces daughter cells with different cell fates (yellow versus green) 
a

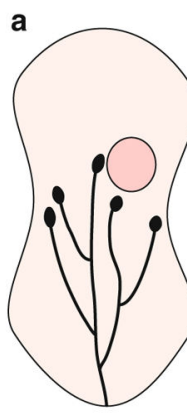

b

b

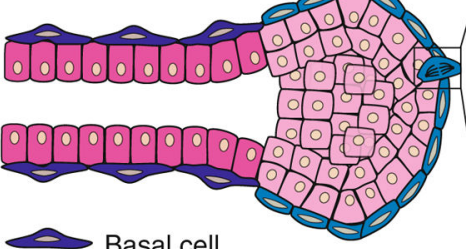

Cap cell

0 Luminal cell

0 Body cell

Fig. 16.6.

SLIT2/ROBO1 signaling in the mammary gland. (a) Cartoon of developing mammary gland. Mammary ducts (black) grow postnatally from the nipple into the fat pad (beige) and at 5.5 weeks reach the lymph node (pink circle). Capping each duct is a terminal end bud, which serves as a site of cell proliferation. (b) Longitudinal section through a terminal end bud and subtending duct. Each duct is bilayered with an outer layer of basal cells and an inner layer of luminal cells. The terminal end bud is a spherical structure with an outer layer of cap cells and inner layers of luminal body cells. (c) SLIT2/ROBO1 signaling in the cap cells of the terminal end bud regulates cell division. In a $W T$ terminal end bud, cap cells undergo ACD and are renewed (blue) while generating a progenitor cell (green). This is due to the expression of INSC (green circles) that accumulates on one side of the dividing cell. Loss of Robo1 ( Robol $^{-/}$) or overexpression of Insc (Insc ${ }^{K I / K I}$ ), both of which increase INSC levels in the cell, results in a switch in division mode from ACD to SCD 